\title{
An enhanced IPv6 anycast routing protocol using Protocol Independent Multicast- Sparse Mode (PIM-SM)
}

\begin{abstract}
Although the demands for finding an economical routing protocol that provides the service to the clients in shortest time and least cost are increasing. IPv6 Anycast still has a lot of issues and problems in practical applications. One of the problems is that IPv6 Anycast still does not have its own standard protocol. In this paper we develop a new Anycast routing protocol by modifying the existing multicast routing protocol because anycast and multicast have many similar properties. Protocol Independent Multicast-Sparse Mode (PIM-SM) is chosen as a basis to design a new anycast routing protocol. We next improve the design by considering the status (free or busy) of the anycast receivers as an important factor in our design. Besides the metric value of the receiver, we propose a new variable in the routing table called BMF (Best Metric Factor).
\end{abstract}

Keyword: BMF (Best Metric Factor); IPv6 anycast routing protocol; Metric; PIM-SM (Protocol Independent Multicast-Sparse Mode) 